\title{
Original
}

\section{Silk Fibroin Regulates Osteoconduction of Hydroxyapatite in Rat Spine Fusion Model}

\author{
Cenhao Wu ${ }^{1)}$, Genlin Wang ${ }^{1)}$, Qi Yan ${ }^{1)}$, Jeffrey C Wang ${ }^{2)}$, Huilin Yang ${ }^{1)}$ and Jun Zou ${ }^{1)}$ \\ 1) Department of Orthopaedic Surgery, The First Affiliated Hospital of Soochow University, Suzhou, Jiangsu, China \\ 2) Department of Orthopaedic Surgery, Keck School of Medicine, University of Southern California, CA, USA \\ (Accepted for publication, August 30, 2019)
}

\begin{abstract}
The purpose of this study was to use a rat spinal fusion model to determine whether osteoconduction of HA is improved by the addition of SF. The SF/HA composite was derived by mixing HA sols with SF sols. In an in vitro study, water absorption testing, cell compatibility and compression test were done. In an in vivo study, $30 \mathrm{~S}-\mathrm{D}$ male rats had surgery for posterolateral lumbar fusion. Group I rats were implanted with SF/HA composites; Group II rats were implanted with HA; Group III rats were implanted with an autograft. Fusion was evaluated by water absorption test, manual palpation, radiology and histology. The results showed that water absorption was significantly higher with the SF/HA composite compared with HA. Manual palpation and radiology showed a higher rate of fusion in the SF/HA group. Histology also showed better results for the SF/HA group. In, conclusion, SF can modify the osteoconduction of HA. SF/HA composites make good bone graft alternatives in spinal fusion, although additional research needs to be done.
\end{abstract}

Key words: Silk fibroin, Hydroxyapatite, Osteoconduction, Spine fusion

\section{Introduction}

As the aging population has caused the higher morbidity of spinal degenerative diseases, the spinal fusion procedures for the treatment of spinal instability or being the next step of decompression of spinal stenosis has drawn a lot of concern. One of the most common fusion procedures is the posterolateral lumbar intertransverse process arthrodesis, which is accomplished through bone graft surgery ${ }^{1)}$. Bone graft surgery has been widely performed in various fields such as orthopaedic, craniomaxillofacial, dental, and plastic surgery.

The use of autogenous sources of bone graft material (often represented by autogenous iliac crest bone graft) for spinal fusion has been considered the "gold standard" for decades because of their osteogenic, osteoconductive and osteoinductive properties. However, there has been a high level of morbidity for complications associated with the harvesting of autogenous bone such as chronic donor site pain, deep infection, vascular injuries, and blood loss at the donor site ${ }^{2,3)}$. The limited volume of autogenous bone is also a disadvantage, especially for patients with osteoporosis or multiple previous bone graft procedures ${ }^{1,4,5)}$. In the meantime, the nonunion rate ranges from $10 \%$ to $40 \%$ along with the increase of fusion levels, which are not so satisfied ${ }^{6-9}$.

Therefore, many researchers have focused on the development of synthetic graft substitutes. Of all bone graft substitutes, Hydroxyapatite (HA) is one of the most popular materials and has been utilized experimentally and clinically in bone graft surgery because it is a biocompatible material with good osteoconductivity ${ }^{10-12)}$. Commercially available forms of hydroxyapatite of natural origin are derived from coral exoskeletons that are composed of calcium carbonate. In spine fusion, the graft environment is significantly different from other fusion sites. The environment is exposed to excessive distraction or tension loads, which

Correspondence to: Dr. Jun Zou, Department of Orthopaedic Surgery, The First Affiliated Hospital of Soochow University, 188 Shizi St. Suzhou, Jiangsu 215006, China; Tel: +86-512-67780111; Email: jzou@suda.edu.cn may cause non-union of the fusion segment. And the grafted materials are surrounded by less osteogenic environments such as muscle and other soft tissues, not bone tissue ${ }^{1,12,13)}$. So the healing capability of the conventional HA is not remarkable in spine fusion, which is often presented as the non-union of the fusion segments. To improve the osteoconduction of HA, many studies try to achieve optimal structural characteristics by changing its porous characteristics or combining it with biocompatible polymers or proteins ${ }^{14-16)}$. Silk fibroin (SF), a hard protein extracted from silk cocoons, is composed of 17 amino acids. SF-based biomaterials has been studied and used for decades as a scaffold for tissue engineering because of its excellent biocompatibility, bioabsorbability, and low level of inflammatory potential ${ }^{17-19)}$. It also has excellent mechanical property and structural initegrity ${ }^{20-23)}$. These properties of SF make SF-based scaffold have the potential to serve as a bone construct which has already attracted many researchers ${ }^{23-25}$. Recently, silk fibers were used as a template for mimicking biomineralization ${ }^{26)}$. It was demonstrated that silk fibers can induce apatite deposition on the surfaces of proteins in a solution mimicking body fluids. That means SF-modified HA nanocomposites can mimic the nanostructure of real bone to some extent because of the ability of SF to induce mineralization, which shows that this nove organic-inorganic hybrid has a potential in the bone tissue engineering. Compounding HA with $\mathrm{SF}$ is, therefore, expected to show enhanced bioaffinity and mechanical toughness as well as improved osteoconductivity. In this study, the ability of silk fibroin to enhance osteoconduction of HA was evaluated in a rat spine fusion model.

\section{Material preparation}

\section{Materials and Methods}

The complex porous materials, SF/HA (obtained from Prof. Mingzhong Li, College of Material Science and Engineering, Soochow University), were prepared using ultrasonic vibration gel method. The procedure was described in our previous paper. Both SF/HA and HA 
materials were cut to $5 \times 5 \times 13 \mathrm{~mm}$ using aseptic technique. Each material was placed in a sterile microfuge tube. These materials were then sterilized by Co 60 .

\section{Water absorption testing}

Water uptake by polymeric implants affects their mechanical properties, degradability, and dimensional stability. Studies have shown that the equilibrium water content and the organization of water within the matrix affect cell adhesion ${ }^{27)}$. Water absorption testing is used to determine the amount of water absorbed under specified conditions. For the water absorption test, SF/HA composites and conventional HA materials were dried in an oven for a specified time and temperature and then placed in a desiccator to cool. Immediately after cooling, the specimens were weighed. The material was then immersed in water at agreed upon conditions, often $23^{\circ} \mathrm{C}$ for 24 hours or until equilibrium was reached. Specimens were removed, patted dry with a lint free cloth, and weighed at 5 different times.

Water absorption (WA) was evaluated according to the following equation:

$\mathrm{WA}(\%)=[(\mathrm{W} 2-\mathrm{W} 1) / \mathrm{W} 1]^{*} 100$

Where W2 is the weight after immersion in water for $24 \mathrm{~h}(\mathrm{~g})$, and $\mathrm{W} 1$ is the oven dry weight before immersion in water $(\mathrm{g})$.

\section{Cell culture and determination of cell compatibility}

Bone marrow stroma cells (BMSCs) are obtained through the filtration and centrifugation of the bone marrow which was harvested from rat's femurs. Sprague Dawley (SD) rats were purchased from Experimental Animal Center of Soochow University (animal production license scxk-0009). Then the cellular suspension was added into the DMEM containing $10 \%$ FBS. BMSCs were cultured in an incubator at $37{ }^{\circ} \mathrm{C}$ with $5 \% \mathrm{CO}_{2}$. When cells reached $90 \%$ confluency, they were passaged at a ratio of 1:2.

Cell compatibility of HA and SF/HA was determined 1 day and 3 days after the BMSCs were inducted into the 2 different solutions containing the 2 kinds of material (HA and SF/HA, both were pre-wetted in consideration of the water absorption, $0.1 \mathrm{~g} / \mathrm{ml}$ ). The measurement procedure was as follows: $10 \mu \mathrm{l}$ of Cell Counting Kit-8 (CCK-8) solution (Dojindo Chemical Technology Co. Ltd, Tokyo, Japan) was added to each well of the 96-well plates (washed twice by preheated PBS) followed by incubation at $37{ }^{\circ} \mathrm{C}$ and $5 \% \mathrm{CO}_{2}$ for $1 \mathrm{~h}$. After incubation, the supernatants were carefully aspirated. To lyse the cells and completely dissolve the precipitates, $100 \mu 1$ dimethyl sulfoxide (DMSO) was added to each well and the plates were shaken for $15 \mathrm{~min}$. The absorbance at $450 \mathrm{~nm}$ was measured and the background absorbance of the medium in the absence of cells was subtracted. The experiments were independently repeated at least three times.

\section{Compression test}

A compression test is used to evaluate the mechanical strength of HA and SF/HA by using a universal mechanical tester (HY-1080, HengYi Precision Instrument Co. Ltd, Shanghai, China) with a load cell at a cross-head which moves at a speed of $0.5 \mathrm{~mm} / \mathrm{min}$. The material was cut into cylinders which were about $6 \mathrm{~mm}$ in diameter and $12 \mathrm{~mm}$ in height. The top and bottom surfaces were polished against 800 -grade sandpaper to ensure they are parallel and smooth. The stress-strain curve was then generated and the force and displacement was recorded until the sample failed. So the maximum strength value before failure was considered as the compressive strength of the sample. Compression test was repeated at least 5 times for each kind of material.

\section{Constructing the L4-L5 posterolateral spine fusion model}

The approval of the animal research committee was obtained prior to the start of animal experiments. Thirty male, Sprague Dawley (SD) rats (purchased from Experimental Animal Center of Soochow University, animal production license scxk-0009) were divided into the following three groups: Group I, SF/HA + autogenous bone; Group II, HA + autogenous bone; Group III, pure autogenous bone only. Rats were anesthetized by isofluorane inhalation and monitored by an assistant during the surgery. A posterior midline incision was made in the skin. Next, two separate paramedian incisions were made $3 \mathrm{~mm}$ from the midline in the lumbar fascia, and the transverse processes of L4 and L5 were exposed. The transverse processes were decorticated with a low-speed burr. Autogenous cancellous bone was harvested from the iliac crest through extended fascial incisions. For groups I-III, the materials and autogenous iliac bone were mixed and grafted to the intertransverse space at L4-5 bilaterally. In the Autogenous group, pure autogenous iliac bone was grafted. Rats were housed in separate cages, allowed to eat and drink ad libitum, and their condition was monitored on a daily basis. At postoperative week eight, all animals were euthanized with intravenous administration of sodium pentobarbital (a $200 \mathrm{mg} / \mathrm{kg}$ bolus) and the lumbar spinal segment from L1 to S2 was removed en bloc with surrounding soft tissue. An operative segment was carefully retrieved and postmortem analyses including biomechanical testing, radiographic and histologic evaluations were performed. All the experimental procedures were approved by the Ethics Committee of the First Affiliated Hospital of Soochow University and were carried out in strict accordance with Declaration of Helsinki (1964) and the Laboratory Animal Guidelines for Ethical Review of Animal Welfare (GB/T35892-2018, China), approval number 378/2017.

\section{Manual assessment of fusion}

Manual palpation has been determined to be the most sensitive and specific method for assessing spinal fusion in this model. Three different blinded observers assessed the spines by manual palpation. After 8 weeks, all spines were explanted and subjected to manual palpation to assess the fusion at L4-L5. The spinal processes of L4 and L5 were located, and then motion was assessed at this level. The spines were graded as either fused or not fused.

\section{Radiographic analysis}

After manual palpation, spines were scanned with high-resolution micro-computerized tomography (micro CT), which utilized the 9-20 $\mu \mathrm{m}$ resolution technology of a $\mu \mathrm{CT} 40$ instrument (Scanco Medical AG Co, Basserdorf, Switzerland). Micro CT data were collected at $55 \mathrm{kVp}$ and $72 \mu \mathrm{A}$ and reconstructed using a cone-beam algorithm supplied with the microCT scanner from Scanco. Visualization and reconstruction of the data were performed using a $\mu \mathrm{CT}$ Ray T3.3 and the $\mu \mathrm{CT}$ Evaluation Program V5.0 (Scanco Medical AG Co, Basserdorf, Switzerland). The reconstructed $3 \mathrm{D}$-images were obtained on the computer to show the fusion condition of the 3 groups.

\section{Histologic analysis}

After sacrifice, spines were dissected, and specimens were fixed in $40 \%$ ethanol, decalcified using standard $10 \%$ decalcifying solution $\mathrm{HCl}$ (Cal-Ex) (Fischer Scientific, Fairlawn, NJ), washed with running tap water, and then transferred to $75 \%$ ethanol. Serial sections near the 
Cenhao Wu et al.: Silk Fibroin Regulates Osteoconduction in Spine Fusion

Table1. Water absorption test

\begin{tabular}{cc}
\hline Group & $\bar{x} \pm \mathrm{s}$ \\
\hline HA $(\mathrm{WA} \%)$ & $13.05 \pm 0.16$ \\
SF/HA $(\mathrm{WA} \%)$ & $73.09 \pm 0.95^{*}$
\end{tabular}

$* \mathrm{P}<0.05$ compared to HA group

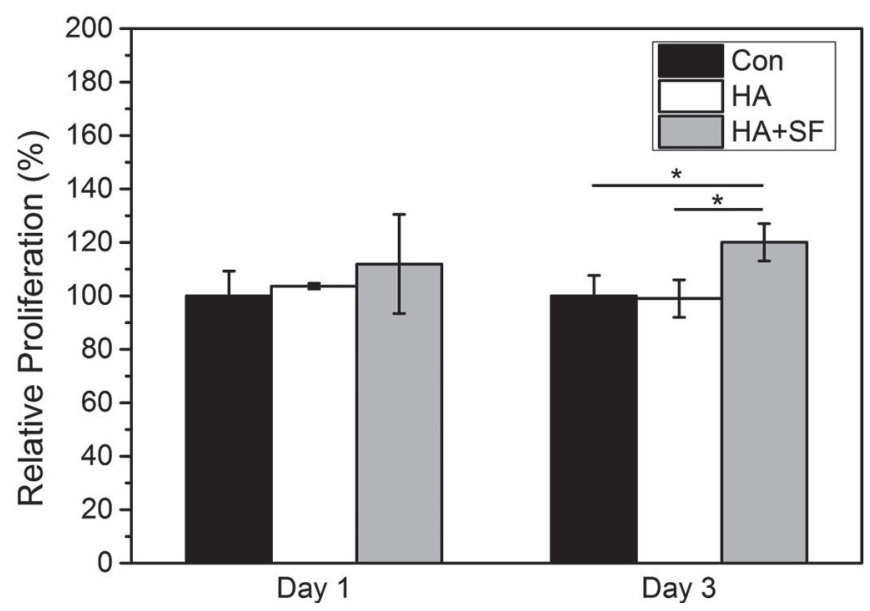

Figure 1. The graph of Cell Counting Kit-8 (CCK8) assay. The Y axis represents the relative proliferation rate. Day 1: One day after BMSCs were cultured with HA and SF/HA, there existed no significant difference in the relative proliferation rate between the control, $\mathrm{HA}$ and $\mathrm{SF} / \mathrm{HA}$ group. Day 3: 3days after the induction, the relative proliferation rate in SF/HA group was significantly higher than that in control and HA group (both $p<0.05$ ), while there was no statistical difference between the control group and HA group.

transverse processes were cut carefully at the level of the transverse process. The specimens were embedded in wax and sectioned. The sections were stained with hematoxylin and eosin.

\section{Statistical methods}

The computer program Statistical Package for the Social Sciences (SPSS) (V13, SPSS, 1 Chicago, IL) was used. Student t-tests were performed when comparing the water absorption (WA), relative cell proliferation and compressive strength between groups. The assessments of solid fusion via manual palpation were compared using chi-square test.

\section{Results}

\section{Water absorption testing}

The water absorbing behavior of conventional HA and SF/HA were investigated and results are shown in Table 1. As SF was increased, water uptake of HA increased. At all times, the water absorption of SF/HA was significantly higher than of HA $(\mathrm{P}<0.05)$. This is primarily due to the presence of improved microstructure of the materials, which facilitates water permeating into the scaffold.

\section{Cell compatibility}

Cell Counting Kit-8 (CCK8) assay (Fig. 1) showed that one day after BMSCs were cultured with $\mathrm{HA}$ and $\mathrm{SF} / \mathrm{HA}$, there existed no significant difference in the relative proliferation rate between the control, HA and SF/HA group. When the time was extended to 3 days after the induction, the relative proliferation rate in SF/HA group was significantly higher than that in control and HA group (both $\mathrm{p}<0.05$ ), while there was no statistical difference between the control group and HA group. This result mainly proved the cell compatibility of the SF/HA hybrid which
Table 2. Solid fusion rate assessed by manual palpation

\begin{tabular}{cccc}
\hline Group & No.Solid Fusion & $\begin{array}{c}\text { No.Non-solid } \\
\text { Fusion }\end{array}$ & $\mathrm{P}$ \\
\hline SF/HA+autograft & 10 & 0 & $* * \mathrm{P}<0.01$ \\
HA+autograft & 5 & 5 & $\mathrm{P}=0.16$ \\
Autograft alone & 2 & 8 & - \\
\hline
\end{tabular}

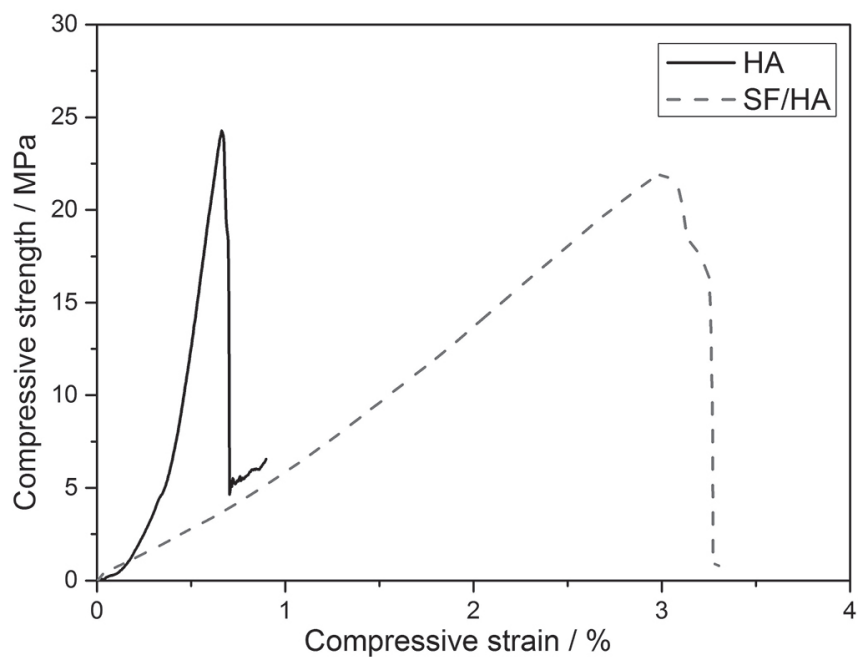

Figure 2. 2 typical stress-strain curve we chosen. Compressive strength of HA: 24.27MPa; Compressive strength of SF/HA: 21.93MPa. The compressive strain of SF/HA is larger than that of HA.

means that this kind of material is not cytotoxic. We also found that the relative proliferation rate in SF/HA group in Day3 was significantly higher, which could be explained by an improvement in the cell microenvironment for the existence of SF. The possible mechanism is discussed later.

\section{Compression test}

We selected 2 typical stress-strain curve in Fig. 2 (Compressive strength of HA: 24.27MPa; Compressive strength of SF/HA: 21.93MPa; the compressive strain of SF/HA is larger than that of HA). The average compressive strength of HA was higher than that of SF/HA. But there were actually no statistical differences between them. (Compressive strength of HA: $23.13 \pm 1.26 \mathrm{MPa}$; Compressive strength of SF/HA: $22.14 \pm 2.15 \mathrm{MPa})$.

\section{Manual assessment of fusion}

Palpation of a continuous bony mass between the intertransverse processes is considered an indication of complete spinal fusion. The proportion of solid-fusion subjects in each group are judged by three independent observers. We defined the "solid bony fusion" as without the ability to generate anterior-posterior or lateral move by manual palpation. The results were shown in Table2. The spines from the SF/HA Group (10/10) had a significantly higher fusion rate than those from the control group $(2 / 10)(\mathrm{p}<0.01)$. Although there were more solid fusion samples in HA group (5/10) compared with the control group, there existed no statistical significance $(\mathrm{p}=0.16)$.

\section{Radiographic analysis}

The three-dimensional CT scan was performed after the rats were sacrificed at 8 weeks. The reconstructed images were seen in Fig. 3. A 
A

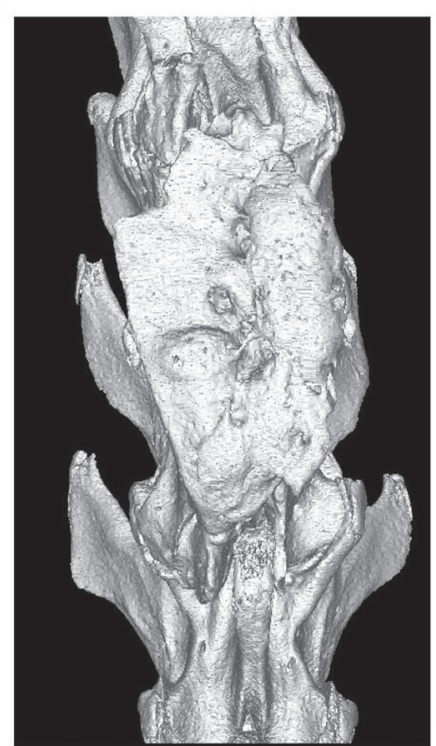

B

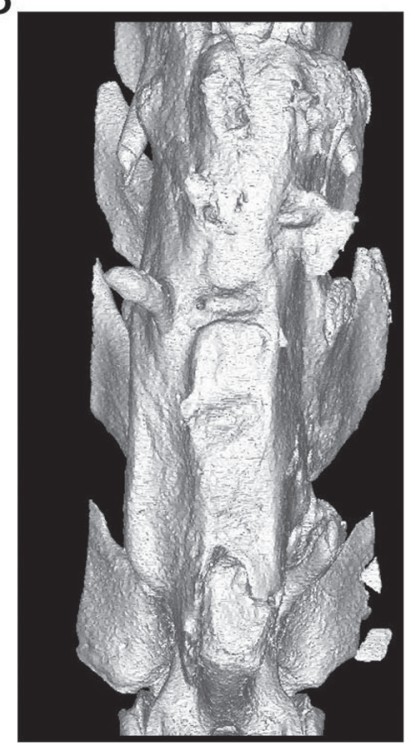

C

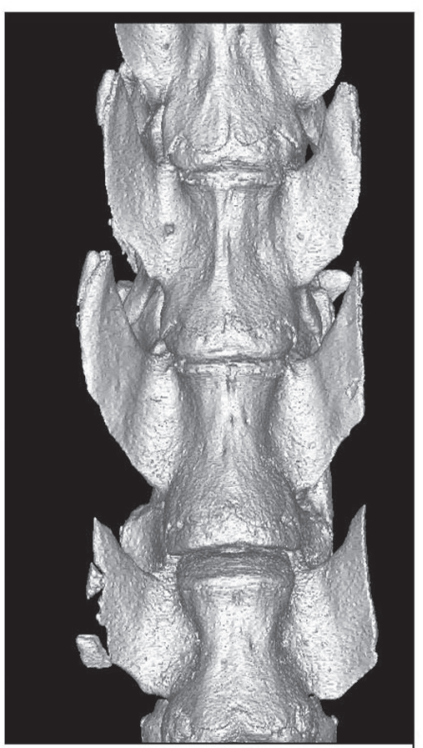

Figure 3. 3D-reconstructed CT images taken at 8 weeks of Sprague Dawley rats that treated with SF/HA+autograft (A), HA+autograft (B) and autograft alone (C). A. A great amount of new bone mass could be seen in the intertransverse and interspinous space in Group I with a very solid fusion. B. Group II also showed the boney bridge between the tranverse process, but the amount of new bone mass was less than that of Group I according to the images. C. The control group showed little or none fusion on the image.

A

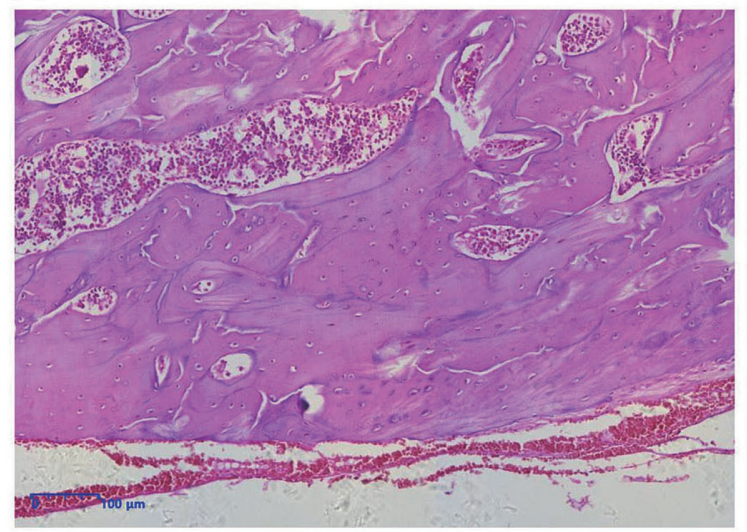

B

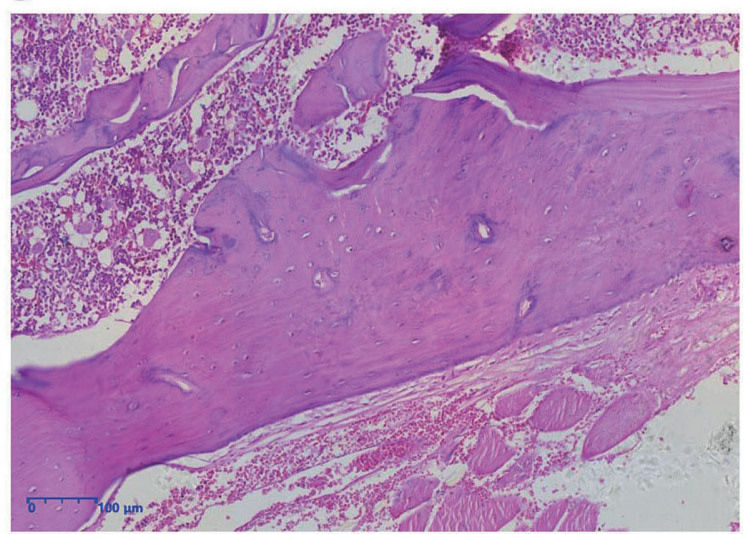

C

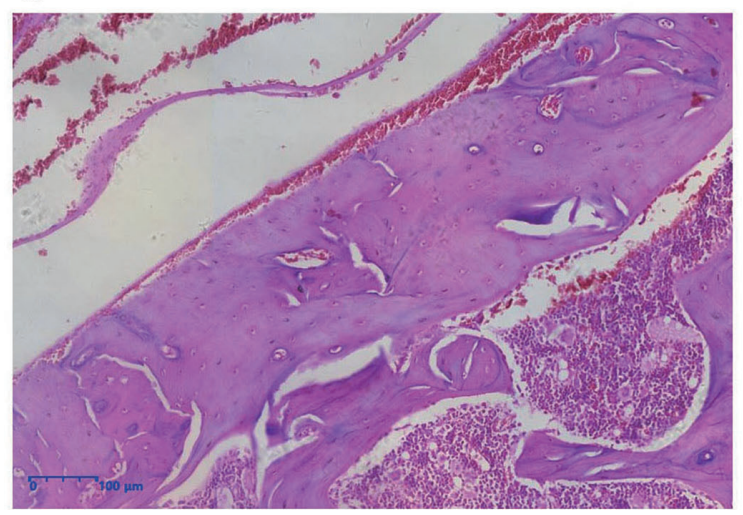

Figure 4. Histological sections (Scale bar $=100 \mu \mathrm{m}$ ) near the transverse processes of Group I (Fig. 4A), Group II (Fig. 4B) and Group III (Fig. 4C). A. Bone ingrowth was achieved throughout the scaffold granules via the interconnecting pores and the area of direct bone bonding was larger than that of the HA groups. The newly formed bone covered the microporous surface of macropores inside the material and the newly formed bone inside the materials remained connected to the bone tissue outside them via the micropores which looks like a relatively homogenous trabecular structure. B. Bone ingrowth could be seen only in peripheral pores of the HA granules, and its surface was mainly covered with fibrous tissue or empty space. Although bridging bone was formed between the L4 and L5 transverse process in these specimens, there was only a little direct bone bonding with the HA granules. C. Samples from autografts (control group) exhibited only a small amount of bone segments between the adjacent transverse processes, which is evidence of incomplete fusion. Fibrous tissue and cartilaginous matrix were also present in the nonunion bridge area. 
great amount of new bone mass could be seen in the intertransverse and interspinous space in Group I with a very solid fusion (Fig. 3A). Group II also showed the boney bridge between the tranverse process (Fig. 3B), but the amount of new bone mass was less than that of Group I according to the images. On the contrary, the control group showed little or none fusion on the image (Fig. 3C).

\section{Histologic examination}

Distinct histological differences were observed between HA and SF/ HA groups according to the histological sections in Fig. 4. For the HA group, bone ingrowth could be seen only in peripheral pores of the HA granules, and its surface was mainly covered with fibrous tissue or empty space. Although bridging bone was formed between the L4 and L5 transverse process in these specimens, there was only a little direct bone bonding with the HA granules (Fig. 4B). On the other hand, for the SF/ HA group, bone ingrowth was achieved throughout the scaffold granules via the interconnecting pores and the area of direct bone bonding was larger than that of the HA groups. For the SF/HA group, SF/HA was integrated with the newly formed bone in the fusion mass. Middle magnification microphotographs of the SH/HA group showed that the newly formed bone covered the microporous surface of macropores inside the material and the newly formed bone inside the materials remained connected to the bone tissue outside them via the micropores which looks like a relatively homogenous trabecular structure (Fig. 4A). High magnification photomicrographs of the SF/HA also showed the presence of osteoblast-like cells attached to the rough surface of the macropores. In contrast to these groups, samples from autografts (control group) exhibited only a small amount of bone segments between the adjacent transverse processes, which is evidence of incomplete fusion. Fibrous tissue and cartilaginous matrix were also present in the nonunion bridge area (Fig. 4C).

\section{Discussion}

The structural differences between $S F / H A$ and $H A$ alone and how the changes improve the osteoconduction

1. SF improves the physicochemical properties of microstructure in the hybrid material

Studies have found that the existence of organic materials in the hybrid structure can regulate the nucleation of inorganic crystals and enhance the physicochemical properties of the microstructure ${ }^{16}$. We have known that SF could promote the preferential growth of HA crystals along the C-axis on an SF matrix. So a highly developed three-dimensional network is built up throughout HA crystals and SF fibrils, leading to an apparent increase in mechanical strength, flexibility and toughness of the composite ${ }^{16}$. It has been proved that the degeree of bone ingrowth and security of fixation is reduced significantly by micromotion between implant and bone ${ }^{28)}$. As the posterior column of spine mainly loads tension or distraction and the lower lumber spine fusion is often characterized by the increased motion in the intertransverse space, the fusion assurance of lumbar is much stricter than that of other area such as the long bone defect. The result of our compression test showed that the SF/ HA had a lager deformation range while didn't decrease the maximum compressive strength. So maybe this property may be more suitable for the mechanical environment in lower lumber spine which may reduce the micromotion between the implant and bone.

\section{SF modulates the porous microstructure of the hybrid material}

Wang et al. found that the different proportion of SF in SF/HA might cause the different porosity, mean pore size and mean compressive strength of the hybrid material ${ }^{29)}$. And at a proper proportion, the following porosity, mean pore size and mechanical strength can cause the most favorable osteoconduction result in his experiment. Actually, previous studies didn't reach an agreement in the optimal geometry for osteoconduction $^{30)}$. They proposed that $\varnothing 100 \mu \mathrm{m}$ sized pore is the smallest pore for osteoconduction and the $\varnothing 500 \mu \mathrm{m}$ sized pore is the optimal pore ${ }^{31}$, ${ }^{32)}$. The latest study indicated that the size of interconnection rather than the porosity or pore size alone could be the key element to determine the degree of conduction despiting the mechanical strength, though the interconnection size is often related to the porosity, pore size, pore shape and mechanical strength in usual ${ }^{30)}$. The histological result of our study indicated that the bone ingrowth was achieved throughout the scaffold granules via the interconnecting pores in SF/HA group, while bone ingrowth could be seen only in peripheral pores of the HA granules in HA group. This has proved that after combined with SF, the interconnection between pores in the SF/HA has already been enhanced, which is beneficial for osteoconduction. In addition, the water absorption test of our study shows that the water absorption of SF/HA was significantly higher than that of HA $(\mathrm{P}<0.05)$. This could be explained by the change of the surface of pores in SF/HA after combining SF with HA. The enhanced water uptake by SF/HA may be related to the rougher surface of the HA pores. The augmentation of the interfacial surface may give more space for cell adhesion, and the enhanced hydrophilicity related to the hydrophilic group of SF means the exchange of substance such as nutrition and cytokines between intracellular fluid and extracellular matrix is more efficient, which is good for cell proliferation and differentiation.

\section{The osteoinductivity of SF/HA given by the silk fibroin}

Previous studies have already found that the silk-based scaffolds provide a suitable substrate for bone marrow mesenchymal stem cells to adhere and proliferate ${ }^{33,34)}$. Recent studies indicated that silk proteins can stimulate osteoblast differentiation by suppressing the Notch signaling pathway in mesenchymal stem cells ${ }^{35}$. So most likely, the silk fibroin gave the osteoinductivity to our SF/HA hybrid. The existence of osteoblast-like cells attached to the rough surface of the macropores in the high magnification photomicrographs of the SF/HA should prove that.

\section{The limitations of $\mathrm{HA}$ in spine fusion}

HA seems to hold promise for use as an extender of autologous iliac crest bone grafting in spinal fusions. Used alone, it has been found to have less than desirable fusion results ${ }^{4}$. HA, as one of the osteoconductive bone graft extenders, essentially improve the bony generation by mimicking the structure of cancellous bone. The bone itself is osteoconductive, especially the cancellous bone, because of its porous and highly interconnected trabecular which makes the in growth of surrounding tissues more easily ${ }^{36)}$. However, when it comes to the spinal fusion, the fusion mechanism varies depending on different regions of the spine. The anterior and middle column of spine are mainly cancellous bone that loads bearing, while the posterior column of spine is mainly the cortical bone and muscular tissue that loads tension or distraction. So the HA alone may not be suitable for the posterolateral spine fusions. Actually there are already some researches show that the pure osteoconductive substitutes are less effective in posterolateral spine fusions than using them in anterior spine ${ }^{1,37)}$.

\section{Blending HA with other polymers for the use in spine fusion}

Attempts have therefore been made to develop alternative composite biomaterials by blending HA with other polymers. It is a great challenge 
to devise and fabricate a new generation of HA-involved composite biomaterials having comparable microstructure and properties with natural bone such as mimicking the inorganic-orgaic composites.

The most common organic material used as bone constructs in spinal fusion is collagen which is one kind of protein polymer that can be obtained from animals or through transgenic techniques. Many researches are working on the composite of HA and collagen. What's more, theses composites can be fabricated into different forms that can often carry some osteoinductive materials like rhBMP-2 (recombinant human bone morphogenetic protein-2) or osteoinductive cells like bone marrow cells ${ }^{15,16,38)}$. All these studies shows that the composite biomaterial can be the substitute for autograft but it's hard to say which kind of composite can be the best for the spinal fusion depending on the fusion rate or stiffness though there may exist histological differences.

Though the collagen is the first choice to mimic the organic phase of natural bone, it still has some limitations such as: it's not cost-effcetive, it may provoke immune response and it retains a risk for contamination $^{39}$. Because of these limitations, studies have been made to blend HA with other natural materials such as silk fibroin.

In conclusion, we tested the ability of SF to enhance osteoconduction of HA in a well-accepted redent spine fusion model. Radiographic results demonstrated both higher rates of fusion and much earlier fusion in the SF/HA group than in the HA or autograft groups. These findings were confirmed by manual palpation and histological examination. Our study indicated that SF/HA worked as a scaffold for newly formed bone, and the trabecular bone both inside and outside the granules firmly connect to each other to provide structural support. On the contrary, the HA group indicated that conventional HA did not integrate with newly formed bone even in fusion masses that obtained solidity. So HA might work only as a space maintainer. The findings of our study provide evidence that it is possible for SF to regulate the osteoconducion of HA, although there is still a lot of work that needs to be done.

\section{Acknowledgements}

This work was supported by National Natural Science Foundation of China (81472132, 81572183 and 91849114), Priority Academic Program Development of Jiangsu Higher Education Institution (PAPD).

\section{Conflict of Interest}

The authors have declared that no COI exists.

\section{References}

1. Boden SD. Overview of the biology of lumbar spine fusion and principles for selecting a bone graft substitute. Spine 27: S26, 2002

2. Summers BN and Eisenstein SM. Donor site pain from the ilium. A complication of lumbar spine fusion. J Bone Joint Surg Br 71: 677680, 1989

3. Younger EM and Chapman MW. Morbidity at bone graft donor sites. J Orthop Trauma 3: 192-195, 1989

4. Spivak JM and Hasharoni A. Use of hydroxyapatite in spine surgery. Eur Spine J 10: S197-S204, 2001

5. Berven S, Tay BK, Kleinstueck FS and Bradford DS. Clinical applications of bone graft substitutes in spine surgery: consideration of mineralized and demineralized preparations and growth factor supplementation. Eur Spine J 10: S169-S177, 2001

6. Bridwell KH, Sedgewick TA, O'Brien MF, Lenke LG and Baldus C. The role of fusion and instrumentation in the treatment of degenerative spondylolisthesis with spinal stenosis. J Spinal Disord Tech 6: 461-472, 1993
7. Fischgrund JS, Mackay M, Herkowitz HN, Brower R, Montgomery DM and Kurz LT. 1997 Volvo Award winner in clinical studies. Degenerative lumbar spondylolisthesis with spinal stenosis: a prospective, randomized study comparing decompressive laminectomy and arthrodesis with and without spinal instrumentation. Spine 22: 2807, 1997

8. France JC, Yaszemski MJ, Lauerman WC, Cain JE, Glover JM, Lawson KJ, Coe JD and Topper SM. A randomized prospective study of posterolateral lumbar fusion. Outcomes with and without pedicle screw instrumentation. Spine 24: 553, 1999

9. Steinmann JC and Herkowitz HN. Pseudarthrosis of the spine. Clin Orthop 284: 494-503, 1992

10. Fujishiro T, Nishikawa T, Niikura T, Takikawa S, Nishiyama T, Mizuno K, Yoshiya S and Kurosaka M. Impaction bone grafting with hydroxyapatite: increased femoral component stability in experiments using Sawbones. Acta Orthop 76: 550-554, 2005

11. Luchetti R. Corrective osteotomy of malunited distal radius fractures using carbonated hydroxyapatite as an alternative to autogenous bone grafting. J Hand Surg 29: 825-834, 2004

12. Matsumine A, Myoui A, Kusuzaki K, Araki N, Seto M, Yoshikawa $\mathrm{H}$ and Uchida A. Calcium hydroxyapatite ceramic implants in bone tumour surgery. A long-term follow-up study. J Bone Joint Surg Br 86: 719-725, 2004

13. Boden SD, Martin GJ, Morone M, Ugbo JL, Titus L and Hutton WC. The use of coralline hydroxyapatite with bone marrow, autogenous bone graft, or osteoinductive bone protein extract for posterolateral lumbar spine fusion. Spine 24: 320-327, 1999

14. Motomiya M, Ito M, Takahata M, Kadoya K, Irie K, Abumi K and Minami A. Effect of hydroxyapatite porous characteristics on healing outcomes in rabbit posterolateral spinal fusion model. Eur Spine J 16: 2215-2224, 2007

15. Akihito M, Munehito Y, Mamoru K, Satoru Y, Hirotsugu K, Hiroshi $\mathrm{H}$ and Boden SD. The use of cultured bone marrow cells in type I collagen gel and porous hydroxyapatite for posterolateral lumbar spine fusion. Spine 30: 1134-1138, 2005

16. Farokhi M, Mottaghitalab F, Samani S, Shokrgozar MA, Kundu SC, Reis RL, Fattahi Y and Kaplan DL. Silk fibroin/hydroxyapatite composites for bone tissue engineering. Biotechnol Adv 36, 2017

17. Altman GH, Diaz F, Jakuba C, Calabro T, Horan RL, Chen J, Lu H, Richmond J and Kaplan DL. Silk-based biomaterials. Biomaterials 24: 401-416, 2003

18. Lorenz M, Sandra H, Vassilis K, Carl KH, John MC, Gloria G, Ludwig Z, Robert L, Gordana VN and Kaplan DL. The inflammatory responses to silk films in vitro and in vivo. Biomaterials 26: 147155,2005

19. Gh PBA, Chen JS, Jin HJ, Karageorgiou V and Kaplan DL. Macrophage responses to silk. Biomaterials 24: 3079-3085, 2003

20. Omenetto FG and Kaplan DL. New opportunities for an ancient material. Science 329: 528-531, 2010

21. Sugihara A, Sugiura K, Morita H, Ninagawa T, Tubouchi K, Tobe R, Izumiya M, Horio T, Abraham NG and Ikehara S. Promotive effects of a silk film on epidermal recovery from full-thickness skin wounds. P Soc Exp Biol Med 225: 58-64, 2010

22. Mottaghitalab F, Farokhi M, Zaminy A, Kokabi M, Soleimani M, Mirahmadi F, Shokrgozar MA and Sadeghizadeh M. A biosynthetic nerve guide conduit based on silk/SWNT/fibronectin nanocomposite for peripheral nerve regeneration. PLoS One 8: e74417, 2013

23. Singh BN, Panda NN, Mund R and Pramanik K. Carboxymethyl cellulose enables silk fibroin nanofibrous scaffold with enhanced bi- 
omimetic potential for bone tissue engineering application. Carbohydr Polym 151: 335-347, 2016

24. Shao W, He J, Han Q, Sang F, Wang Q, Chen L, Cui S and Ding B. A biomimetic multilayer nanofiber fabric fabricated by electrospinning and textile technology from polylactic acid and Tussah silk fibroin as a scaffold for bone tissue engineering. Mater Sci Eng C Mater Biol Appl 67: 599-610, 2016

25. Bhattacharjee P, Naskar D, Maiti TK, Bhattacharya D, Das P, Nandi SK and Kundu SC. Potential of non-mulberry silk protein fibroin blended and grafted poly( $($-caprolactone) nanofibrous matrices for in vivo bone regeneration. Colloid Surface B 143: 431-439, 2016

26. Takeuchi A, Ohtsuki C, Miyazaki T, Ogata S, Tanihara M, Tanaka H, Furutani $\mathrm{Y}$ and Kinoshita H. Apatite formation on silk fiber in a solution mimicking body fluid. Key Eng Mater 240: 31-34, 2003

27. Scotchford CA, Sim B, Downes S and Braden M. Water uptake and protein release characteristics of a new methacrylate-based polymer system. Polymer 38: 3869-3874, 1997

28. Jasty M, Bragdon C, Burke D, O'Connor D, Lowenstein J and Harris WH. In vivo skeletal responses to porous-surfaced implants subjected to small induced motions. J Bone Joint Surg Am 79: 707, 1997

29. Wang G, Yang H, Li M, Lu S, Chen X and Cai X. The use of silk fibroin/hydroxyapatite composite co-cultured with rabbit bone-marrow stromal cells in the healing of a segmental bone defect. J Bone Joint Surg Br 92: 320-325, 2010

30. Chang BS, Lee iCKfC-K, Hong KS, Youn HJ, Ryu HS, Chung SS and Park KW. Osteoconduction at porous hydroxyapatite with various pore configurations. Biomaterials 21: 1291-1298, 2000

31. Flatley TJ, Lynch KL and Benson M. Tissue response to implants of calcium phosphate ceramic in the rabbit spine. Clin Orthop Relat $\mathrm{R}$ 179: 246,1983

32. Hulbert SF, Morrison SJ and Klawitter JJ. Tissue reaction to three ceramics of porous and non-porous structures. J Biomed Mater Res 6: 347,2010

33. Mandal BB and Kundu SC. Cell proliferation and migration in silk fibroin 3D scaffolds. Biomaterials 30: 2956-2965, 2009

34. Mauney JR, Nguyen T, Gillen K, Kirker-Head C, Gimble JM and Kaplan DL. Engineering adipose-like tissue in vitro and in vivo utilizing human bone marrow and adipose-derived mesenchymal stem cells with silk fibroin 3D scaffolds. Biomaterials 28: 5280-5290, 2007

35. Jung SR, Song NJ, Dong KY, Cho YJ, Kim BJ, Hong JW, Yun UJ, Jo DG, Lee YM and Choi SY. Silk proteins stimulate osteoblast differentiation by suppressing the Notch signaling pathway in mesenchymal stem cells. Nutr Res 33: 162-170, 2013

36. Cornell $\mathrm{CN}$ and Lane JM. Current understanding of osteoconduction in bone regeneration. Clin Orthop Relat R 355: S267, 1998

37. Acharya NK, Kumar RJ, Hari Krishna V and Menon VK. Hydroxyapatite-bioactive glass ceramic composite as stand-alone graft substitute for posterolateral fusion of lumbar spine: a prospective, matched, and controlled study. J Spinal Disord Tech 21: 106-111, 2008

38. Tay BK, Le AX, Heilman M, Lotz J and Bradford DS. Use of a collagen-hydroxyapatite matrix in spinal fusion. A rabbit model. Spine 23: 2276-2281, 1998

39. Chunling DU, Jin J, Yucheng LI, Kong X, Wei K and Yao J. Novel silk fibroin/hydroxyapatite composite films : Structure and properties. Mat Sci Eng C-Mater 29: 62-68, 2009 
J.Hard Tissue Biology Vol. 28(4): 341-348, 2019 